\title{
COMPUTATIONAL ANALYSIS OF HOMEODOMAIN-LEUCINE ZIPPER (HD-ZIP) FAMILY OF TRANSCRIPTION FACTORS IN ARABIDOPSIS THALIANA
}

\author{
M. S. Javaid, U. A. Ashfaq, M. Ijaz, B. Naz, B. Saleem, A. Arshad and Mahmood-ur-Rahman* \\ Department of Bioinformatics \& Biotechnology, GC University, Faisalabad-38000, Pakistan \\ ${ }^{*}$ Corresponding Author's email: mahmoodansari@gcuf.edu.pk
}

\begin{abstract}
Homeodomain-leucine Zipper (HD-Zip) proteins are a class of transcription factors having conserved homeodomain. Homeo box, conserved part of homeodomain consists of 56 residues and is involved in plant development. We retrieved protein sequences of all the $48 \mathrm{HD}$-Zip family members, performed genome-wide analysis, and grouped them into four subfamilies. A comprehensive genomic analysis including mapping of all the transcription factors on 5 chromosomes of Arabidopsis thaliana, multiple sequence alignment to find out the conserved domains, phylogenetic, and gene structure analysis by mapping introns and exons, promoter analysis by taking $1000 \mathrm{bp}$ upstream genomic sequence of all these transcription factors and motif analysis was performed in this study. The results helped us to understand the functional homology among HD-Zip transcription factors, comparison of the tree with motifs that further depends upon the number of exons and introns. This classification and analysis further categorized the transcription factors of 4 HD-Zip subfamilies into different classes and revealed a deep evolutionary relationship among them and thus helped to further explore their functioning.
\end{abstract}

Keywords: HD-Zip family, Arabidopsis thaliana, Homeo box, phylogenetic analysis, motif

https://doi.org/10.36899/JAPS.2021.4.0312

Published online December 18, 2020

\section{INTRODUCTION}

Many biological processes such as development, growth, cell division and response against stress or environmental stimuli in organisms/cells are controlled by transcription factors (Shen et al., 2019). Transcription factors (TFs) bind to the DNA and either up-regulate or down-regulate gene expression at the transcriptional level (Todaka et al., 2015). There are more than 60 families of TFs in plants having different functions. The homeo-box (HB) family of TFs has a sub-family of homeodomainleucine-zipper (HD-Zip) proteins that have been reported in Arabidopsis thaliana and many other plants. The HB is an important transcription factor family consisting of the homeodomain (HD) which binds to DNA at a specific site and a leucine zipper that acts as a dimerization motif (Wang and Zhuang, 2019). The HD-Zip proteins play fundamental roles in various plant developmental processes, from cell formation pattern to specifications of cell types (Shao et al., 2018). The HD-containing proteins were grouped into six classes including KNOX (KNOTTED1-like homeobox), HD-Zip (homeodomainleucine-zipper), Bell (bell domain), PHD-Finger (plant homeodomain-finger), ZF-HD (zinc fingerhomeodomain) and WOX (Wuschel-related homeobox). Out of these classes, the HD-Zip proteins are abundant in plants and perform important functions in different processes of development and growth (Chen et al., 2014).

In Arabidopsis, the HD-Zip family has 48 members that are further divided into 4 sub-families based on their biological roles and other conserved motifs (Gong et al., 2019). HD-Zip I, II, III and IV proteins preferentially bind with the $\operatorname{CAAT}(\mathrm{A} / \mathrm{T}) \mathrm{ATTG}$, CAAT(C/G)ATTG, GTAAT(G/C)ATTAC and CATT(A/T)AATG motifs respectively (Sessa et al., 2018). The HD-Zip family has a highly conserved HDdomain and a less conserved leucine zipper (LZ) motif and four sub-families are differentiated on the basis of downstream sequence of LZ domain (Ding et al., 2017). LZ domain is a dimmer of Basic-region leucine zipper (bZip) class of TFs (Vinson et al., 1989). The bZip domain is 60-80 amino acids in length with conserved DNA binding basic-region and a diversified LZ region (Zhang et al., 2014). The localization of the leucines is very important for the DNA binding to the proteins. In Arabidopsis, the HD-Zip I subfamily is comprised of 17 candidate genes that do not show response to ABA signaling, sugar signaling and environmental stresses but are crucial for plant de-etiolation and embryogenesis (Henriksson et al., 2005). The HD-Zip II subfamily of Arabidopsis has 9 members and all of them consist of a cellular redox status perceptive CPSCE motif located in the downstream region of $\mathrm{LZ}$ domain and most of these genes respond to shading, light and auxin as disclosed by biochemical and genetic analyses (Turchi et al., 2013).

HD-Zip III has 5 TFs that are basically regulatory elements, involved in various developmental processes like apical embryo development, the formation of shoot meristematic tissues, determining the polarity of different organs, vascular diversity and transference of auxins 
(Wenkel et al., 2007). The fourth sub-family, HD-Zip IV, also known as HD-G12 is a larger one and contains 16 candidate genes. The genetic analysis illustrated that proteins belong to this subfamily play critical roles in the formation of the trichome, root propagation, accumulation of anthocyanin and differentiation of epidermal cells in Arabidopsis (Chen et al., 2017). The HD-Zip family has been reported in many studies and considerable work has been done on its sub-families, but the focus was not driven on genome-wide analysis. The purpose of this research was to study 48 HD-Zip TFs, their phylogenetic analysis, conserved domain analysis, promoter analysis, gene structure analysis, analysis of cis-regulatory elements and chromosomal mapping by using various bioinformatics tools in Arabidopsis to understand their functional dynamics.

\section{MATERIALS AND METHODS}

Chromosome mapping: The physical location of TFs of the HD-Zip family was demonstrated in A. thaliana. The chromosomal mapping of all the 48 members of the HDZip family on the different chromosomes of Arabidopsis was performed by the online tool, TAIR (http://www.arabidopsis.org/jsp/ChromosomeMap/tool.js p) by using standard procedures.

Identification of conserved domains: The core of the Homeo-domain was studied in all the 48 genes of the HDZip family of $A$. thaliana including all the subfamilies. For this purpose, multiple sequence alignment of all the members was performed by Unipro UGENE v. 34 (http://ugene.net/) (Rose et al., 2018). Then protein sequences of all the candidate genes were compared for identification of the whole domain.

Phylogenetic analysis: The phylogenetic analysis and multiple sequence alignment (MSA) of protein sequences of HD-Zip genes were performed by using MEGA 6.0 software (https://www.megasoftware.net/). HD-Zip genes were grouped into different sub-families depending upon their biological functions and domain structure. The parameters used for alignment were as follow, penalty for gap opening: 10; penalty for gap extension: 0.2 ; weight matrix for protein: gonnet; penalties for specific residues: on; hydrophilic penalties: on; separation distance for gap: 4; end of gap separation: off; negative matrix usage: off; cut-off for delay divergent: $30 \%$. An un-rooted phylogenetic tree was built by neighbor-joining (NJ) method by using multiple sequence alignment.

Synteny analysis: The protein sequences of all the HDZip genes were retrieved from an online database of plant transcriptional factors (http://planttfdb.cbi.pku.edu.cn/) for Synteny analysis. The sequences were saved in the FASTA format. Then, Synteny analysis was performed to identify the conservation of homologous genes by using an online tool by following default parameters (http://tools.bat.infspire.org/circoletto/).

Gene structure analysis: TAIR (https://www.arabidopsis.org/) and "Database for Transcription Factors of Arabidopsis" (DATF) (http://datf.cbi.pku.edu.cn/) were used to fetch the information and annotated the gene structure, e.g. number of exons and introns. The Microsoft PowerPoint and excel sheets were used to analyse the gene structure.

Analysis of conserved motifs: MEME 4.9.1 software (http://meme.nbcr.net/meme/cgi-bin/meme.cgi) was used for the identification of conserved motifs in different protein domains. The complete dataset (protein sequences) of 48 TFs of HD-Zip was copied in corresponding box having features/properties to specify the results were; number of repetitions - any, number of repetitions, number of motifs -15 , minimum motif width -10 , maximum motif width-56.

Promoter analysis and determination of cis-regulatory elements: Promoter analysis was done by retrieving $1000 \mathrm{bp}$ upstream region of the transcription initiation site of all 48 genes from Phytozome 9.1 database (http://www.phytozome.net/). A complete list of cis-acting elements was obtained by using PLACE, an online tool (Higo et al., 1999) (http://www.dna.affrc.go.jp/PLACE/). We chose common cis-regulatory elements in promoter sequences of all HD-ZIP genes. The location of cisregulatory elements was mapped by using Microsoft PowerPoint.

\section{RESULTS AND DISCUSSION}

Chromosomal mapping: The expression of HD-Zip gene family is highly regulated and shows response to environmental stimuli, organ-specific signals, to light, and to the stress conditions. All candidates of HD-Zip family were examined for the presence of $\mathrm{LZ}$ and $\mathrm{HD}$ domain. This family is further sub-divided into four sub-families HD-Zip I to IV. The first and forth sub-family have high number of genes while second family has a minimum number of genes and third family also have very few genes in Arabidopsis (Sessa et al., 2018). We retrieved 48 candidate genes of HD-Zip TFs present in Arabidopsis. Different variants of these TFs were excluded (only those TFs were studied that have " 1 " value after decimal point e.g. AT3G01470.1). They were mapped on all the 5 chromosomes of $A$. thaliana (Figure-1) using an online chromosomal mapping software from TAIR (http://www.arabidopsis.org/jsp/ChromosomeMap/tool.js p) database. First chromosome had 11 genes where 6 and 5 genes were present on short and long arms, respectively. The second chromosome contained 9 genes; 1 and 8 genes on short and long arms, respectively. The third chromosome had 6 genes; having equal numbers of genes 
on both arms. The fourth and fifth chromosomes had 11 genes where forth chromosome have 2 genes on its short arm and fifth chromosome has 4 genes on its short arm while 9 and 7 genes are present on the long arm of fourth and fifth chromosome, respectively (Figure-1).

Depending upon the information retrieved through 'Phytozome', the chromosomal location of all the 48 genes belonging to HD-Zip family of TFs was determined. The genes of HD-Zip family of TFs were randomly dispersed on all of the 5 chromosomes of $A$. thaliana. It was found that 11 genes of HD-Zip family were located on chromosome number 1, 4 and 5 each while 9 genes were present on chromosome number 2 . Moreover, 6 genes were found on chromosome number 3 . The above data indicated the uneven distribution of HDZip genes on Arabidopsis chromosomes (Figure-1). Our results showed the same pattern as of the distribution of Trithorax homolog genes in chickpea (Qasim et al., 2018), WRKY TFs in Arabidopsis (Sultan et al., 2016), Potassium transporter genes in chickpea (Azeem et al., 2018) and WRKY TFs in chickpea (Waqas et al., 2019).
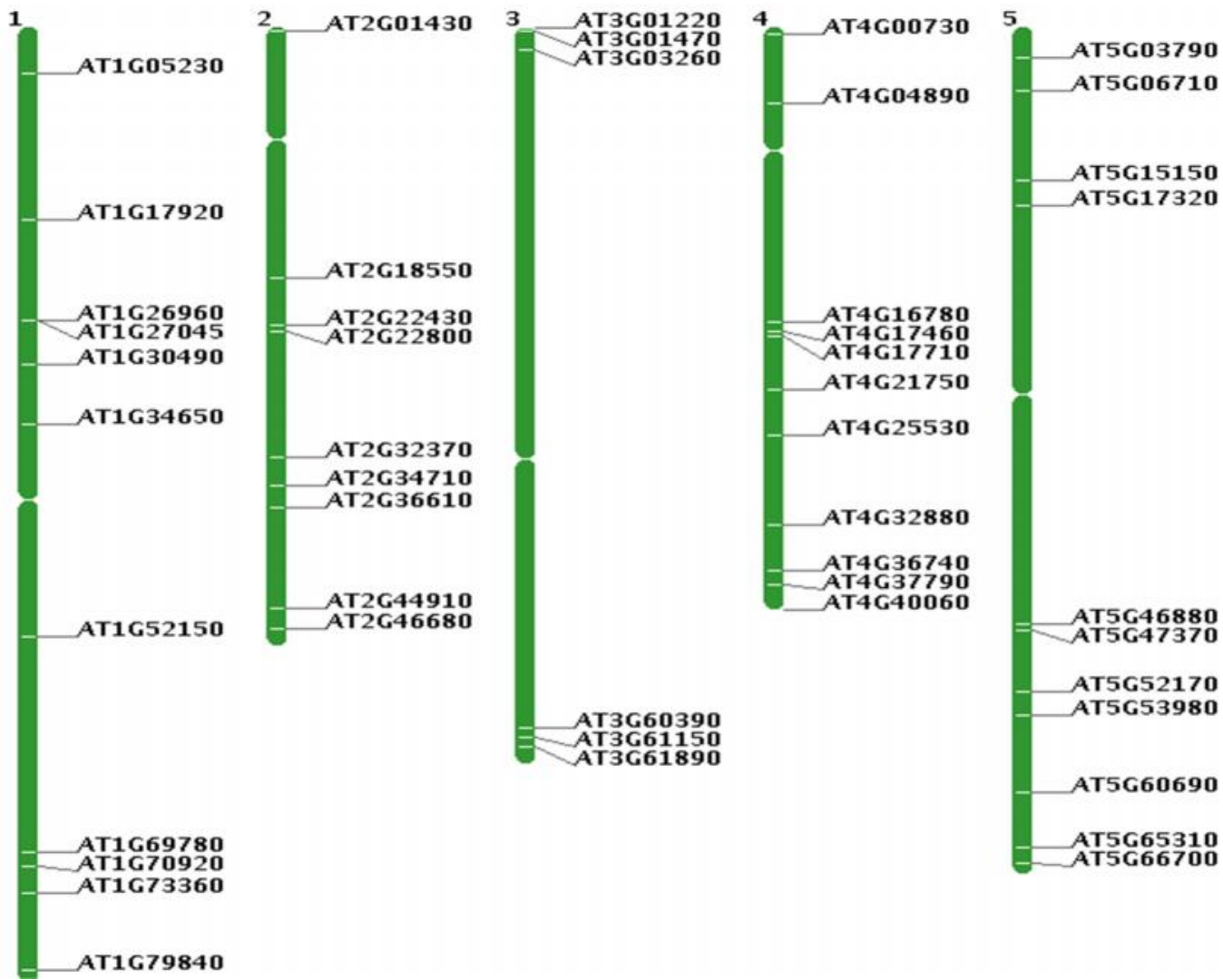

Figure-1: The mapping of HD-Zip transcription factors on $A$. thaliana chromosomes. The numbers of chromosomes are shown at the top of each chromosome and transcription factors are mapped according to their positions. The length of each chromosome is indicating its size.

Identification of conserved domain: HD-Zip family members of Arabidopsis were aligned by using Unipro UGENE software and analysed for the conserved domain. The signature box named "WFQNrr" was identified by UGENE in which "WFQN" residues were highly conserved while "rr" residues were semi-conserved. When protein sequences of all the 48 genes of this family were compared for the identification of whole domain, the total of 44 residues before the WFQNrr and 6 residues after the WFQNrr were identified as conserved with few alternative residues in some genes. So, the Homeo-domain of 56 residues was identified (Figure-2). The conserved amino acids were not present on the same position in all genes.
They were present in different positions but found as a box which shows presence of amino acids as a conserved box in the domain in all the genes.

Moreover, 44 residues at upstream side and 6 residues at downstream side of this box were identified as conserved in all genes with few exceptions and thus it was investigated that the complete domain was present in distributed form and on different positions in every gene. So, to draw a consensus sequence of the domain of all the genes as well as to identify the complete domain as a whole, we deleted the amino acid residues one by one from both; the upstream and the downstream side of WFQNrr from each gene to bring this box on the same position in 
every gene. By this, WFQNrr were placed on the same position in every gene when there were only 44 residues before this box and 6 residues after this box. Thus, Homeo- domain of 56 residues was identified in all genes having WFQNrr amino acids on position number 45, 46, 47, 48, 49 and 50 respectively (Figure 2 ).

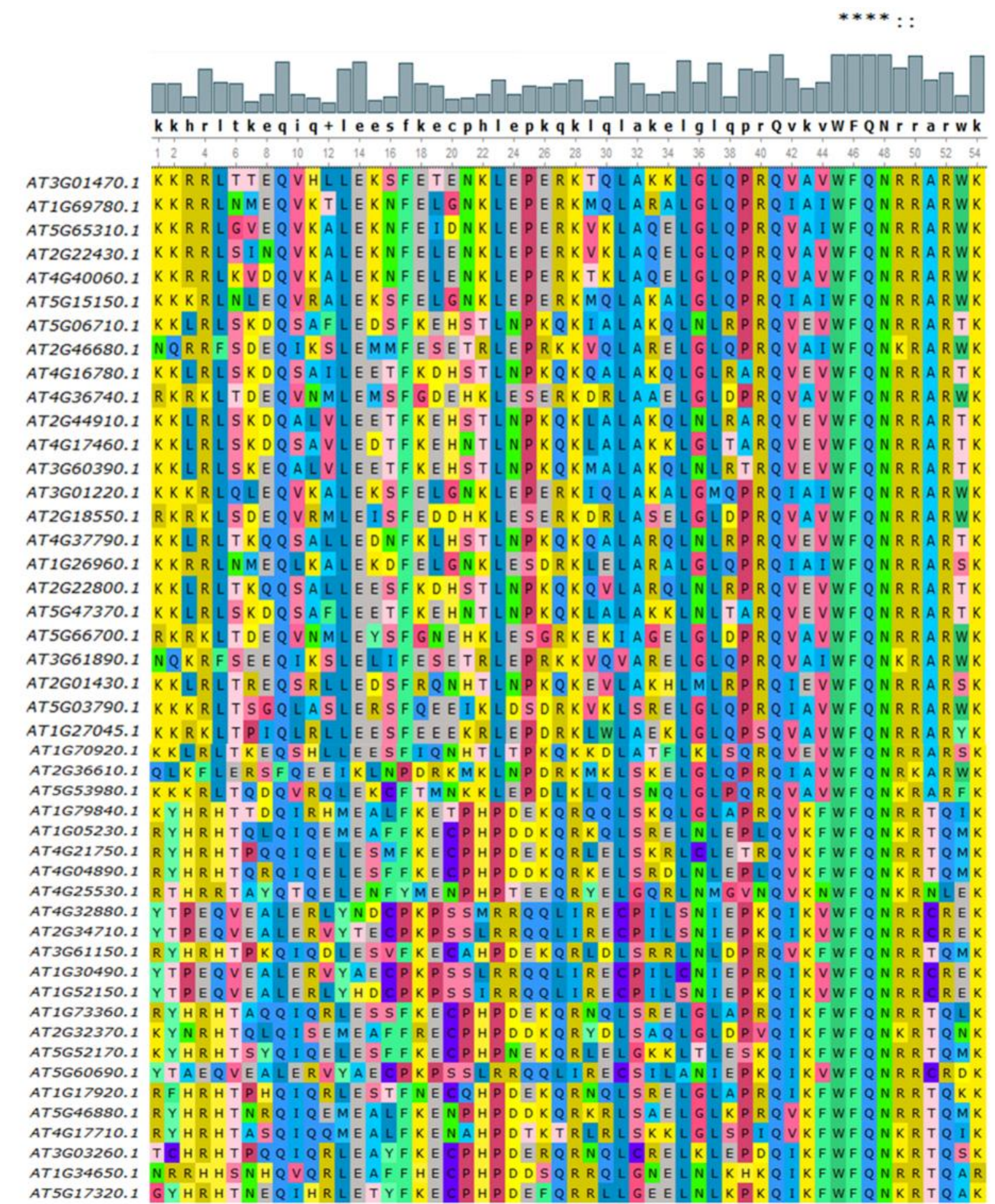

Figure-2: Multiple sequence alignment of HD-Zip transcription factors of $A$. thaliana by Unipro UGENE v. 34 software to identify the conserved domain. The signature sequence WFQNrr is identified and amino acids are shown in figure by " *" and ": " shape.

The proteins of HD-Zip I subfamily were identified via existence of homeo-domain which was closely related to leucine zipper motif. It was found in many studies that some proteins of the first sub-family 
were intricated to the sucrose signalling and abscisic acid pathways. These proteins also played a critical role in the abiotic stress responses of the plants and cotyledon as well as involved in the leaf development and embryogenesis (Valdes et al., 2012; Sessa et al., 2018). Most of the genes of HD-Zip II sub-family were incriminated in phytochrome-mediated organ development e.g. leaf morphogenesis. Some genes were also responsive to the changes of light quality and auxin and shade avoidance as exhibit by biochemical and genetic analyses (Turchi et al., 2013, 2015).

Phylogenetic analysis: A total of 4 sub families were observed in a phylogenetic tree. All the 4 sub families had conserved homeo-domain. HD-Zip subfamily I, II, III and IV have 17, 10, 5 and 16 genes respectively and each gene in each subfamily was categorized in different clades/classes according to their ancestor and evolutionary relationship as well as functions. It was concluded from the phylogenetic analysis that specific subfamilies of HDZip played specific role in specific type of responses in growth and development of $A$. thaliana. For phylogenetic analysis, an un-rooted tree was generated from all the 48 genes of HD-Zip members. First sub-family of HD-Zip TFs was divided into 7 clades that were named as clade alpha $(\alpha)$, beta one $(\beta 1)$, beta two $(\beta 2)$, phi $(\Phi)$, epsilon $(\varepsilon)$, gamma $(\gamma)$ and delta $(\delta)$. The HD-Zip II subfamily has 10 members that were divided into 5 clades and named as clade $\alpha, \beta, \varepsilon, \gamma$ and $\delta$ (Figure-3). Proteins of all the members of this family have a conserved set of cysteine residue on inner side and a leucine zipper motif at the outer side.

The HD-Zip III and IV subfamilies were characterized with the help of 2 more conserved domains; first domain was steroidogenic-acute-regulatory-protein related lipid-transfer (START) and second domain was START-adjacent-domain (SAD). Both subfamilies were differentiated by a fifth domain that was the C-terminalMEKHLA-motif (Zhang et al., 2014). This domain was present in third subfamily of HD-Zip proteins, but it was absent in forth subfamily. The third subfamily has 5 members that were divided into 3 clades and named as clade $\alpha, \beta$ and $\gamma$. The HD-Zip IV subfamily has 16 members that were divided into 6 clades and named as alpha $(\alpha)$, beta $(\beta)$, epsilon $(\varepsilon)$, gamma $(\gamma)$, delta $(\delta)$ and zeta $(\zeta)$ (Figure-3).
Members of third sub-family were the key developmental regulators for the Arabidopsis shoot radial patterning and apical embryo. They were also involved in the development of lateral organ polarity, formation of shoot meristem, vascular differentiation and transportation of auxin (Qiu et al., 2006). The proteins of the HD-Zip IV subfamily were involved to play role in epidermal processes, formation of trichome, accumulation of anthocyanin and development of root (Roodbarkelari and Groot, 2017). It helps to understand the evolutionary relationship among all the genes in more detail as each gene is further classified into different clades (Sessa et al., 2018). This may help in further functional and comparative annotation of all the transcription factors of HD-Zip family.

Comparison of HD-Zip Genomic Sequences: Synteny relationship among all the 48 transcription factors of HDZip family of $A$. thaliana was observed in a circular figure as colour bars. Synteny analysis revealed tandem duplication and segmental duplication events in most of the transcription factors within the HD-Zip family. It was observed that transcription factors AT5G17320.1, AT5G52170.1, AT3G03260.1, AT2G32370.1, AT1G34650.1, AT1G52150.1, 0890 and 0333 have no synteny relationship at all. It was confirmed by synteny analysis of HD-Zip family where duplication events were shown by colour ribbons. Transcription factor "AT5G53980.1" that fall in $\Phi$ clade of HD-Zip I subfamily has been observed to play a prominent role in these duplication events. It was also observed that some transcription factors were not involved in segmental and tandem duplication events and thus these factors have their unique sequences and belonged to HD-Zip III and IV subfamilies (Figure-4).

Synteny analysis showed the tandem, whole genome and segmental duplication events that have significance in the evolution of many organisms. Genome duplication events played a significant role in the expansion of family (Hofberger et al., 2015) and in Arabidopsis there are three duplication events. Based on the comprehensive analysis of chromosomal mapping, structure analysis of genes, phylogenetic analysis and analysis of motifs, we observed segmental and tandem duplication in most of the transcription factors of all the 4 subfamilies of HD-Zip transcription factors family. 


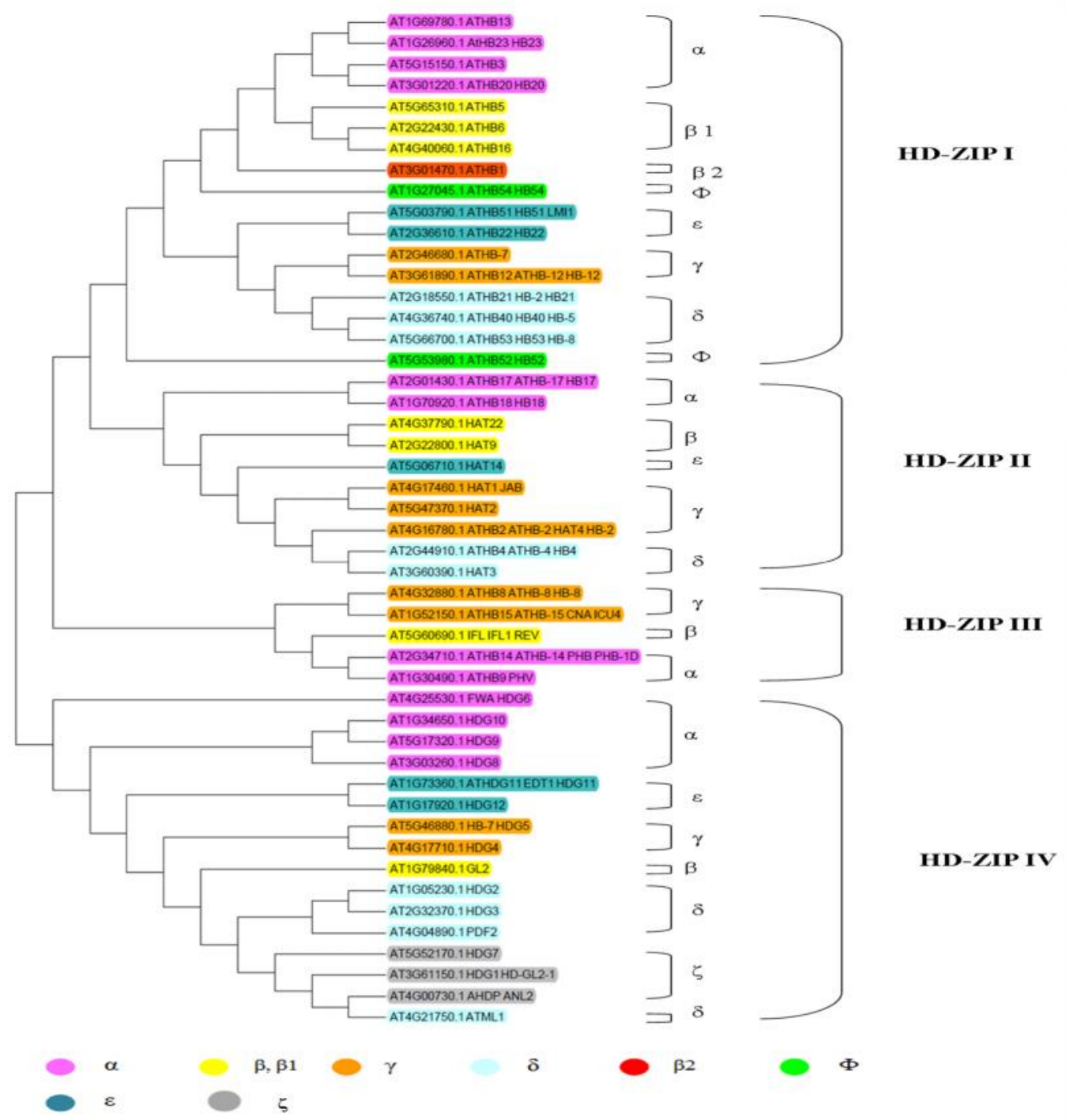

Figure-3: Rectangular phylogenetic tree of HD-Zip family of $A$. thaliana indicating the division of HD-Zip family into four sub families on the basis of evolution and functions.

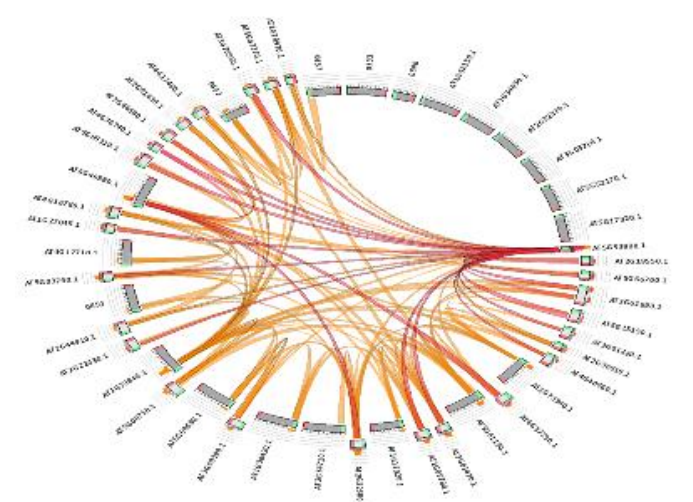

Figure-4: Comparison of gene sequences and identification of homologs of HD-Zip transcription factors by Synteny analysis in $A$. thaliana
Analysis of cis-regulatory elements in promoter region: Transcription factors regulate the expression of genes under biotic and abiotic stress conditions. The first step for promoter analysis was the retrieval of $1000 \mathrm{bp}$ upstream to the start codon for each gene of HD-Zip family of $A$. thaliana. Then data were put into the PLACE database to find out the transcription factor binding sites (motifs) in the promoter region (Supplementary Table-1). We selected five most abundant cis-regulatory elements for each nucleotide sequence in all members of HD-Zip. Three out of five cis-regulatory elements named as CCAATBOX1, WORKY710S \& DOFCOREZM were found in whole family, where they were present on positive strand of DNA. GATABOX was present almost $85 \%$ in promoter region of 48 genes while there was variability at $5^{\text {th }}$ cis-regulatory element (Figure-5), which was mostly present on negative strand of DNA. 


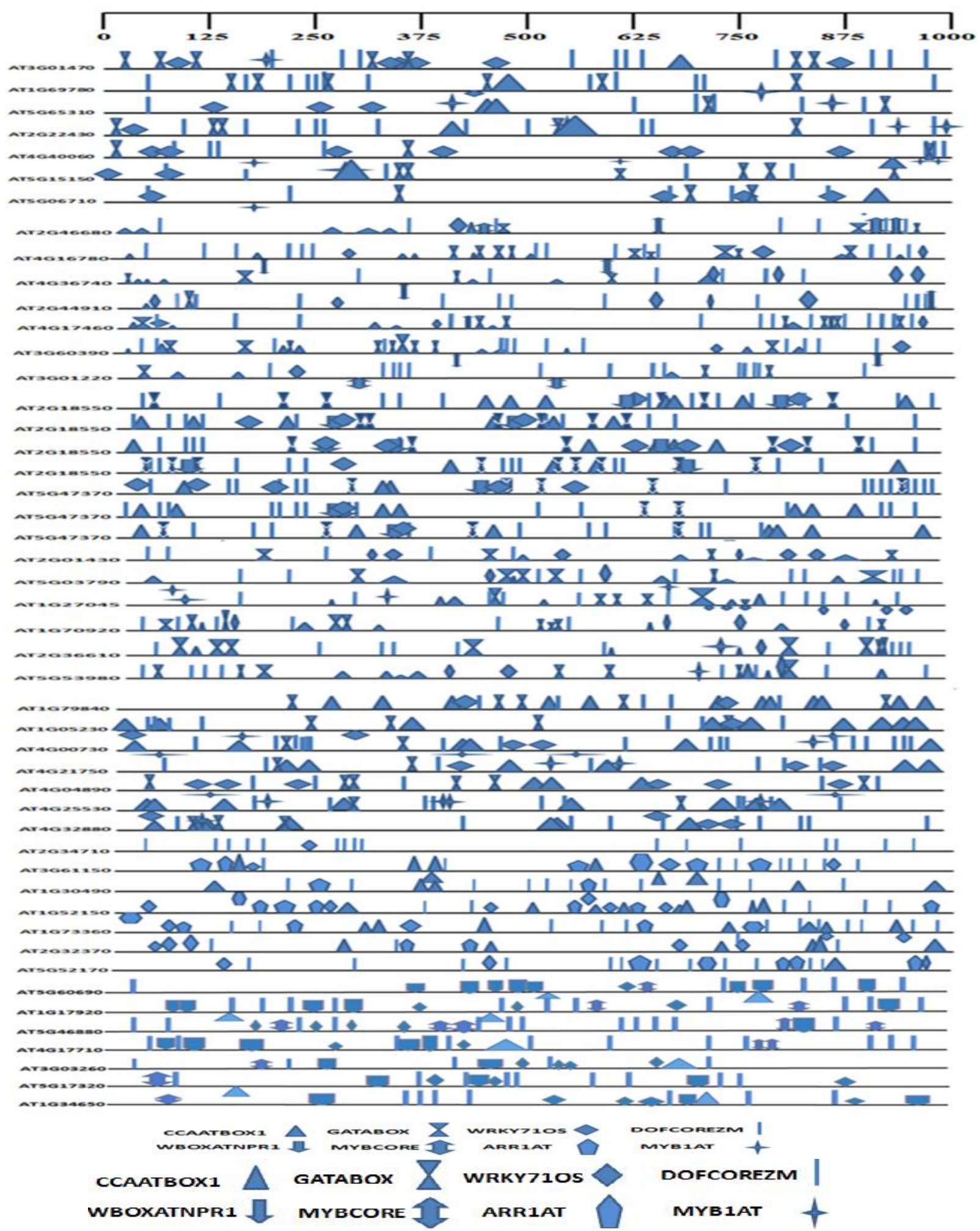

Figure-5: Promoter analysis; schematic representation of various PLACE-based motifs in $1 \mathrm{~kb}$ promoter region of HD-Zip family. Most of motifs were present on + strand as shown in the figure. The motif codes and respective sequences are indicated in the figure. 
CCAATBOX1, WORKY710S, GATABOX \& DOFCOREZM were selected for analysis of cisregulatory elements. CCAATBOX1 with site CCAAT was present in all members of HD-Zip family. The CCAAT Box regulates the flowering of A. thaliana and is mostly present in non-coding region of heat shock proteins of eukaryotes. DOFCOREZM with site AAAG involved in tissue specific expression. WORKY710S is a stress responsive cis-regulatory element and it was found in whole family of Arabidopsis thaliana. Promoter analysis of cis-regulatory element had revealed that almost every gene contains repetitive binding sites of transcriptional factors on the promoter region (Figure-5).

Identification of conserved motifs: Conserved motif analysis has revealed the homology between sub-families of HD-Zip TFs. As sub-families I and II have same number of motifs and exons while, sub-families III and IV have same number of motifs and exons. Thus, besides the phylogenetic analysis, motif analysis also showed the evolutionary relationship between the HD-Zip subfamilies. Motif 1 links all the sub-families as it is conserved in all the transcription factors of all 4 subfamilies (Figure-6). Based on presence of motifs, we concluded that genes belong to I and II family of HD-Zip TFs were closely related while members of subfamily III and IV also had homology. Motif 1 was conserved in 47 members. Majority of members of this family almost first 28 members were containing 3-4 exons and few of remaining members containing 7-10 exons while there were 2-3 exceptional genes containing 17-18 exons which is showing their evolutionary relatedness and differences (Figure-6).
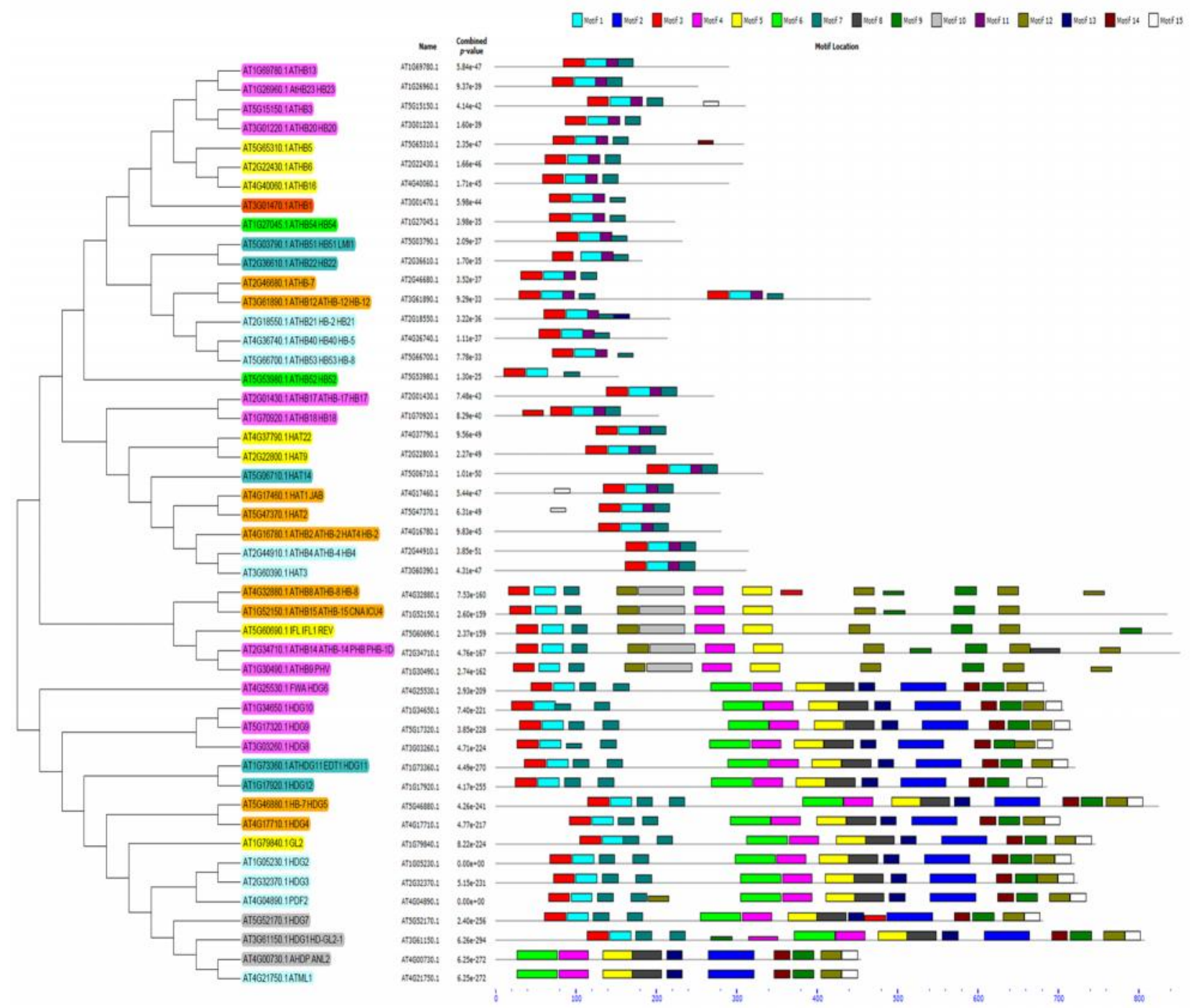

Figure-6: Phylogenetic relationship and motif composition; Conserved motif analysis done by using MEME online software and tree was constructed by using MEGA 6.0 software through multiple sequence alignment. 
Analysis of gene Structure (Exons and Introns): Analysis of gene structure was used for mapping of exons, introns and UTR regions on the gene and thus to find out the location. These regions especially the coding regions that could be further used for functional annotation. The structural analysis of HD-Zip genes was done to find evidence of evolution from this family in $A$. thaliana. The observations revealed that number of exons was 1 to 18 in each gene. AT2G46680.1, AT3G61890.1, AT5G53980.1 had just one exon, while there were three genes that contain 18 exons including: AT1G30490.1, AT1G52150.1 \& AT5G60690.1. The conserved number of introns and exons in genes was an indication for evolutionary relatedness (Figure-7).

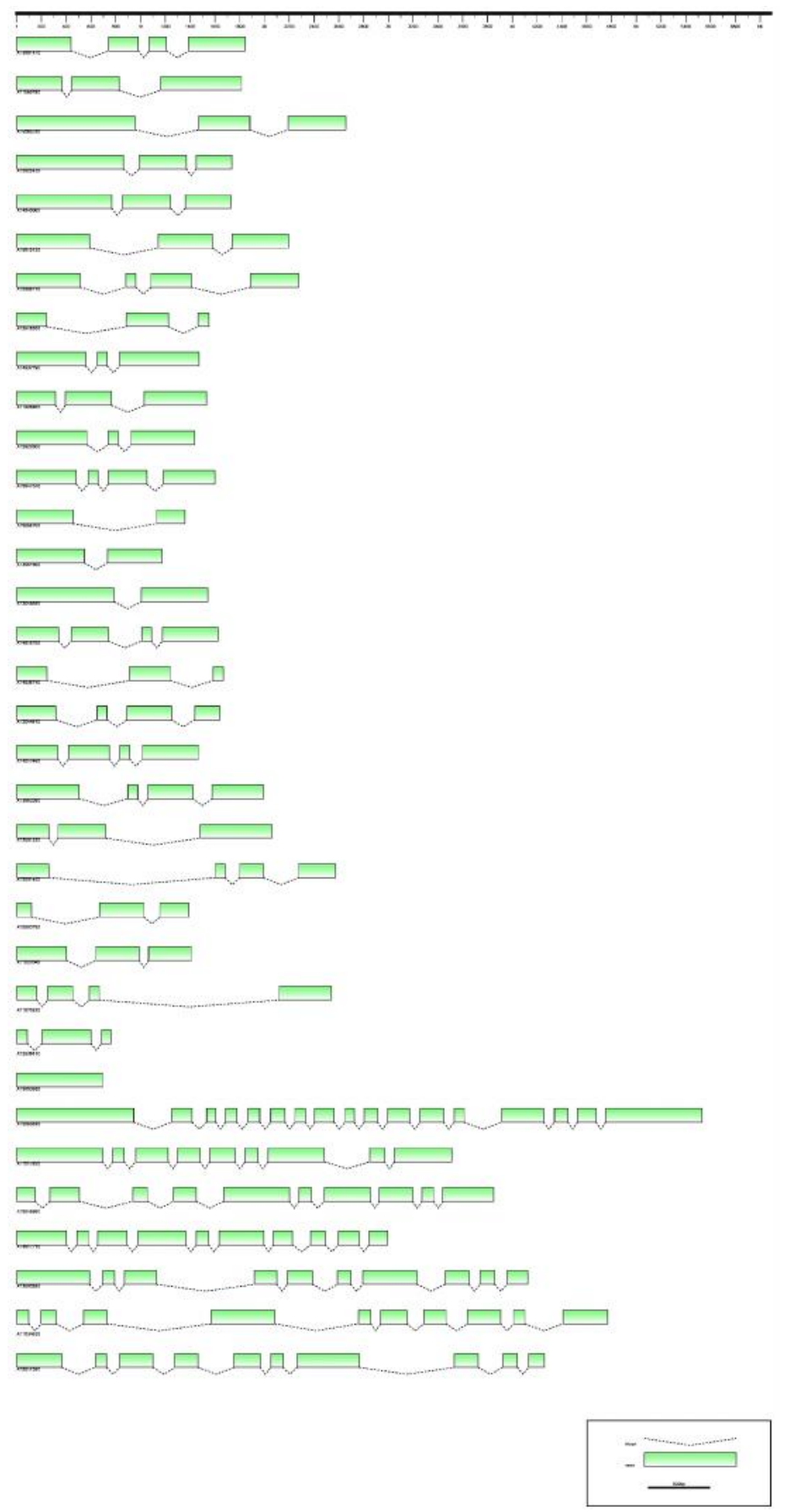

Figure-7: Gene structure analysis; Exon/intron structures of HD-Zip genes in A. thaliana. Colored boxes showing exons while line between boxes representing introns. Location of intron/exon could be estimated by using scale on the top. 
Gene structure analysis revealed similarities and differences between genes of HD-Zip family to explain their evolutionary relationship. According to phylogenetic analysis this family was further divided in 4 sub-families depending upon the functional and structural similarities so to validate that fact we compared the results of structure analysis of genes, phylogenetic analysis and conserved motif analysis. In the result of comparison, majority of members of HD-Zip subfamily I and II were rich in few motifs like motif $1,3,11$ and 15 while there were few exceptions that contain repeats of these motifs. Members of HD-Zip family III had 10 out of 15 motifs and members of HD-Zip family IV were composed of almost all 15 motifs which were selected to perform conserved analysis.

Conclusion: Here in the present study, we presented computational analysis of HD-Zip transcription factors family of $A$. thaliana, providing information about chromosomal mapping to locate the genes on chromosomes, motif analysis and analysis of conserved domains. The analysis of conserved domains represented that 44 residues were conserved before the signature box WFQNrr and 6 residues were conserved after the WFQNrr with few alternative residues in some genes, a total of 4 sub families were observed in the phylogenetic analysis. All the 4 sub families had conserved homeobox domain. This classification and analysis further categorized the transcription factors of 4 HD-Zip subfamilies into different clades and revealed a deeper evolutionary relationship among them. Thus, it may help to explore further functions of these transcription factors. The structural analysis of genes was performed for mapping location of exons and introns in gene. Promoter analysis of cis-regulatory element had revealed that almost every gene contains repetitive binding sites for transcriptional factors on the promoter region.

Acknowledgements: The authors gratefully acknowledge the financial support from Higher Education Commission of Pakistan through a research project grant.

\section{REFERENCES}

Azeem, F., B. Ahmad, R.M. Atif, M.A. Ali, H. Nadeem, S. Hussain, H. Manzoor, M. Azeem and M. Afzal (2018). Genome-Wide Analysis of Potassium Transport-Related Genes in Chickpea (Cicer arietinum L.) and Their Role in Abiotic Stress Responses. Plant Mol. Biol. Rep. 36:451-468.

Chen, E., X. Zhang, Z. Yang, X. Wang, Z. Yang, C. Zhang, Z. Wu, D. Kong, Z. Liu, G. Zhao, H. I. Butt, X. Zhang and F. Li (2017). Genome-wide analysis of the HD-ZIP IV transcription factor family in Gossypium arboreum and GaHDG11 involved in osmotic tolerance in transgenic Arabidopsis. Mol. Genet. Genome 292:593-609.

Chen, X., Z. Chen, H. Zhao, Y. Zhao, B. Cheng and Y.
Xiang (2014). Genome-wide analysis of soybean HD-Zip gene family and expression profiling under salinity and drought treatments. PloS One 9:e87156.

Ding, Z., L. Fu, Y. Yan, W. Tie, Z. Xia, W. Wang, M. Peng, W. Hu and J. Zhang (2017). Genome-wide characterization and expression profiling of HDZip gene family related to abiotic stress in cassava. PloS One 12:e0173043.

Gong, S., Y. Ding, S. Hu, L. Ding, Z. Chen and C. Zhu (2019). The role of HD-Zip class I transcription factors in plant response to abiotic stresses. Physiol. Plant. 167:516-525.

Henriksson, E., A. S. B. Olsson, H. Johannesson, H. Johansson, J. Hanson, P. Engström and E. Söderman (2005). Homeodomain leucine zipper class I genes in Arabidopsis. Expression patterns and phylogenetic relationships. Plant Physiol. 139:509-518.

Higo, K., Y. Ugawa, M. Iwamoto and T. Korenaga (1999). Plant cis-acting regulatory DNA elements (PLACE) database: 1999. Nucleic acids Res. 27:297-300.

Hofberger, J. A., D.L. Nsibo, F. Govers, K. Bouwmeester and M.E. Schranz (2015). A complex interplay of tandem-and whole-genome duplication drives expansion of the L-type lectin receptor kinase gene family in the brassicaceae. Genome Biol. Evol. 7:720-734.

Qasim, M., M. Azeem, M. Zubair, U. Qaiser, A.A. Shah, H. Nadeem, H. Manzoor, S. Hussain, B. Ahmad and F. Azeem (2018). Phylogenetic Analyses of the Trithorax Homologs and Related Proteins in Cicer arietinum. Int. J. Agric. Biol. 20:575-582.

Qiu, C. X., K.J. Zuo and K.X. Tang (2006). Class III Homeodomaiii-leuciiie Zipper Genes in Plant Development. Pakistan J. Biol. Sci. 9: 568-573.

Roodbarkelari, F. and E.P. Groot (2017). Regulatory function of homeodomain-leucine zipper (HDZIP) family proteins during embryogenesis. New Phytol. 213:95-104.

Rose, R., O. Golosova, D. Sukhomlinov, A. Tiunov and M. Prosperi (2018). Flexible design of multiple metagenomics classification pipelines with UGENE. Bioinformatics, bty901.

Sessa, G., M. Carabelli, M. Possenti, G. Morelli and I. Ruberti (2018). Multiple links between HD-Zip proteins and hormone networks. Int. J. Mol. Sci. 19:e4047.

Shao, J., I. Haider, L. Xiong, X. Zhu, R.M.F. Hussain, E. Övernäs, A.H. Meijer, G. Zhang, M. Wang, H.J. Boumeestr and P.B.F. Ouwerkerk (2018). Functional analysis of the HD-Zip transcription factor genes Oshox 12 and Oshox14 in rice. PloS One 13:e0199248.

Shen, W., H. Li, R. Teng, Y. Wang, W. Wang and J. 
Zhuang (2019). Genomic and transcriptomic analyses of HD-Zip family transcription factors and their responses to abiotic stress in tea plant (Camellia sinensis). Genomics 111:1142-1151.

Sultan, S., M.A. Ali, R.M. Atif, F. Azeem, H. Nadeem, M.H. Siddique, E. Filiz, K. Hussain and A. Abbas (2016). Genome wide analysis of stress responsive WRKY transcription factors in Arabidopsis thaliana. Turk. J. Agric-Food Sci. Technol. 4:279-290.

Todaka, D., K. Shinozaki and K. Yamaguchi-Shinozaki (2015). Recent advances in the dissection of drought-stress regulatory networks and strategies for development of drought-tolerant transgenic rice plants. Front. Plant Sci. 6:1-20.

Turchi, L., S. Baima, G. Morelli and I. Ruberti (2015). Interplay of HD-Zip II and III transcription factors in auxin-regulated plant development. J. Exp. Bot. 66:5043-5053.

Turchi, L., M. Carabelli,V. Ruzza,M. Possenti,M. Sassi,A. Peñalosa,G. Sessa,S. Salvi,V. Forte,G. Morelli and I. Ruberti (2013). Arabidopsis HD-Zip II transcription factors control apical embryo development and meristem function. Development 140:2118-2129.

Valdes, A.E., E. Övernäs, H. Johansson, A.R. Iglesias and P. Engström (2012). The homeodomain-leucine zipper (HD-Zip) class I transcription factors ATHB7 and ATHB12 modulate abscisic acid signalling by regulating protein phosphatase $2 \mathrm{C}$ and abscisic acid receptor gene activities. Plant Mol. Biol. 80:405-418.

Vinson, C.R., P.B. Sigler and S.L. McKnight (1989). Scissors-grip model for DNA recognition by a family of leucine zipper proteins. Sci. 246:911916.

Waqas, M., M.T. Azhar, I.A. Rana, F. Azeem, M.A. Ali, M.A. Nawaz, G. Chung and R.M. Atif (2019). Genome-wide identification and expression analyses of WRKY transcription factor family members from chickpea (Cicer arietinum L.) reveal their role in abiotic stress-responses. Genes \& genomics, 41:467-481.

Wang, J. and L. Zhuang (2019). Identi fi cation and characterization of novel homeodomain leucine zipper ( HD-Zip ) transcription factors associated with heat tolerance in perennial ryegrass. Environ. Exp. Bot. 160:1-11.

Wenkel, S., J. Emery, B.H. Hou, M.M.S. Evans and M.K. Barton (2007) A feedback regulatory module formed by LITTLE ZIPPER and HD-ZIPIII genes. The Plant Cell 19:3379-3390.

Zhang, C.H., R.J. Ma, Z.J. Shen, X. Sun, N.K. Korir and M.L. Yu (2014). Genome-wide analysis of the homeodomain-leucine zipper (HD-ZIP) gene family in peach (Prunus persica). Genet. Mol. Res, 13:2654-2668.

Supplementary Table 1: Regulatory elements present in all the $48 \mathrm{HD}-\mathrm{Zip}$ TFs and their locations on promoter.

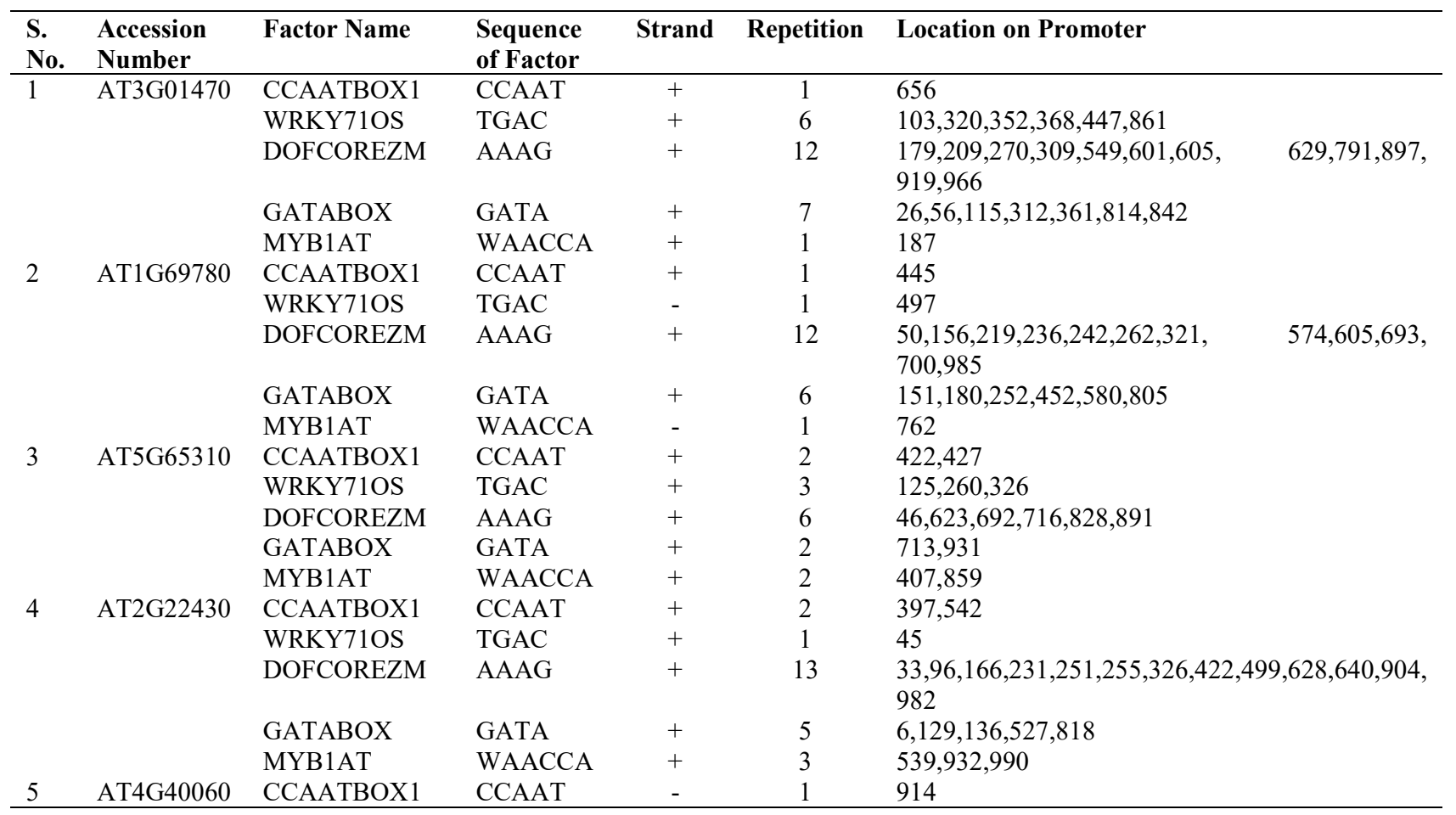




\begin{tabular}{|c|c|c|c|c|c|c|}
\hline & & WRKY71OS & TGAC & + & 7 & $49,77,281,377,675,687,856$ \\
\hline & & DOFCOREZM & AAAG & + & 7 & $83,127,140,262,972,976,997$ \\
\hline & & GATABOX & GATA & + & 3 & $10,268,979$ \\
\hline & & MYB1AT & WAACCA & - & 4 & $170,618,962,987$ \\
\hline \multirow[t]{5}{*}{6} & AT5G15150 & CCAATBOX1 & CCAAT & + & 1 & 277 \\
\hline & & WRKY71OS & TGAC & + & 2 & 2,88 \\
\hline & & DOFCOREZM & AAAG & + & 5 & $65,158,339,681,813$ \\
\hline & & GATABOX & GATA & + & 6 & $357,378,610,753,793,919$ \\
\hline & & MYB1AT & WAACCA & + & 1 & 274 \\
\hline \multirow[t]{5}{*}{7} & AT5G06710 & CCAATBOX1 & CCAAT & + & 1 & 912 \\
\hline & & WRKY71OS & TGAC & + & 4 & $74,651,752,870$ \\
\hline & & DOFCOREZM & AAAG & + & 5 & $53,217,667,736,861$ \\
\hline & & GATABOX & GATA & + & 3 & $339,700,764$ \\
\hline & & MYB1AT & WAACCA & - & 1 & 173 \\
\hline \multirow[t]{5}{*}{8} & AT2G46680 & CAATBOX1 & CAAT & + & 6 & $11,19,252,284,298,413$ \\
\hline & & WRKY71OS & TGAC & + & 1 & 384 \\
\hline & & DOFCOREZM & AAAG & + & 7 & $83,319,452,797,808,972,983$ \\
\hline & & GATABOX & GATA & + & 3 & $476,878,969$ \\
\hline & & MYBCORE & CNGTTR & + & 1 & 667 \\
\hline \multirow[t]{5}{*}{9} & AT4G16780 & CAATBOX1 & CAAT & + & 10 & $39,173,320,326,371,440,123,596,855,962$ \\
\hline & & WRKY71OS & TGAC & + & 4 & $261,558,770,976$ \\
\hline & & DOFCOREZM & AAAG & + & 14 & $\begin{array}{l}77,126,158,228,234,244,510 \\
534,635,668,678,911,919,954\end{array}$ \\
\hline & & GATABOX & GATA & + & 9 & $389,407,486,492,640,691,750,758,870$ \\
\hline & & MYBCORE & CNGTTR & - & 2 & 178,606 \\
\hline \multirow[t]{5}{*}{10} & AT4G36740 & CAATBOX1 & CAAT & + & 6 & $129,148,159,426,581,724$ \\
\hline & & WRKY71OS & TGAC & + & 4 & $727,797,920,955$ \\
\hline & & DOFCOREZM & AAAG & + & 6 & $280,464,697,737,789,801$ \\
\hline & & GATABOX & GATA & + & 4 & $22,169,362,633$ \\
\hline & & MYBCORE & CNGTTR & - & 1 & 342 \\
\hline \multirow[t]{5}{*}{11} & AT2G44910 & CAATBOX1 & CAAT & + & 1 & 64 \\
\hline & & WRKY71OS & TGAC & + & 5 & $97,253,664,738,818$ \\
\hline & & DOFCOREZM & AAAG & + & 11 & $103,132,235,399,414,456,611,762,954,974,979$ \\
\hline & & GATABOX & GATA & + & 1 & 119 \\
\hline & & MYBCORE & CNGTTR & + & 1 & 989 \\
\hline \multirow[t]{4}{*}{12} & AT4G17460 & CAATBOX1 & CAAT & + & 6 & $14,118,273,327,462,817$ \\
\hline & & WRKY71OS & TGAC & + & 3 & $66,352,958$ \\
\hline & & DOFCOREZM & AAAG & + & 15 & $\begin{array}{l}97,155,209,364,418,704,788 \\
798,835,850,869,875,887,926\end{array}$ \\
\hline & & $\begin{array}{l}\text { GATABOX } \\
\text { MYBCORE }\end{array}$ & GATA & + & 7 & $38,447,480,791,801,853,906$ \\
\hline \multirow[t]{5}{*}{13} & AT3G60390 & CAATBOX1 & CAAT & + & 9 & $15,149,243,279,423,487,572,756,825$ \\
\hline & & WRKY71OS & TGAC & + & 2 & 746,968 \\
\hline & & DOFCOREZM & AAAG & + & 13 & $\begin{array}{l}38,49,205,263,449,467,482,515,609,783,842,858 \\
905\end{array}$ \\
\hline & & GATABOX & GATA & + & 9 & $120,163,229,274,290,313,348,353,776$ \\
\hline & & MYBCORE & CNGTTR & - & 2 & 431,953 \\
\hline \multirow[t]{5}{*}{14} & AT3G220 & CAATBOX1 & CAAT & + & 3 & $113,160,673$ \\
\hline & & WRKY71OS & TGAC & + & 1 & 222 \\
\hline & & DOFCOREZM & AAAG & + & 13 & $\begin{array}{l}193,302,320,335,341,548,641 \\
685,736,744,757,763,892\end{array}$ \\
\hline & & GATABOX & GATA & + & 3 & $77,721,787$ \\
\hline & & MYBCORE & CNGTTR & - & 2 & 580,264 \\
\hline \multirow[t]{5}{*}{15.} & AT2G18550 & CAATBOX1 & CAAT & + & 7 & $436,460,515,668,678,760,941$ \\
\hline & & WBOXATNPR1 & TTGAC & + & 2 & 625,848 \\
\hline & & DOFCOREZM & AAAG & + & 12 & $\begin{array}{l}89,125,332,351,388,630,653, \quad 704,775,802,918, \\
990\end{array}$ \\
\hline & & GATABOX & GATA & + & 6 & $59,206,250,639,694,874$ \\
\hline & & WRKY71OS & TGAC & + & 2 & 626,849 \\
\hline \multirow[t]{3}{*}{16.} & AT4G37790 & CAATBOX1 & CAAT & + & 6 & $46,119,209,419,560,613$ \\
\hline & & WBOXATNPR1 & TTGAC & + & 2 & 260,498 \\
\hline & & DOFCOREZM & AAAG & + & 11 & $33,75,89,191,235,556,576,634,646,879,942$ \\
\hline
\end{tabular}




\begin{tabular}{|c|c|c|c|c|c|c|}
\hline \multirow{5}{*}{17.} & \multirow{5}{*}{ AT1G26960 } & GATABOX & GATA & + & 6 & $275,289,459,519,579,620$ \\
\hline & & WRKY71OS & TGAC & + & 3 & $159,261,499$ \\
\hline & & CAATBOX1 & CAAT & + & 5 & $23,573,641,657,712$ \\
\hline & & WBOXATNPR1 & TTGAC & + & 3 & $252,301,666$ \\
\hline & & DOFCOREZM & AAAG & + & 8 & $59,85,104,115,358,455,928,950$ \\
\hline \multirow{6}{*}{18.} & \multirow{5}{*}{ AT2G22800 } & GATABOX & GATA & + & 6 & $210,374,588,791,844,888$ \\
\hline & & WRKY71OS & TGAC & + & 5 & $253,302,626,667,784$ \\
\hline & & CAATBOX1 & CAAT & + & 4 & $382,529,598,939$ \\
\hline & & WBOXATNPR1 & TTGAC & - & 1 & 767 \\
\hline & & DOFCOREZM & AAAG & + & 12 & $58,64,132,207,238,404,429,459,612,623,793,851$ \\
\hline & \multirow{5}{*}{ AT5G47370 } & GATABOX & GATA & + & 9 & $71,76,118,396,547,562,602,657,662$ \\
\hline \multirow{3}{*}{19.} & & WRKY71OS & TGAC & + & 1 & 261 \\
\hline & & CAATBOX1 & CAAT & + & 3 & $101,351,359$ \\
\hline & & WBOXATNPR1 & TTGAC & + & 2 & 96,449 \\
\hline \multirow{6}{*}{20.} & & DOFCOREZM & AAAG & + & 16 & $\begin{array}{l}71,132,139,154,183,215,744 \\
888,894,916,925,929,940,947,971,993\end{array}$ \\
\hline & \multirow{5}{*}{ AT5G66700 } & GATABOX & GATA & + & 5 & $235,481,509,614,968$ \\
\hline & & WRKY71OS & TGAC & + & 5 & $35,97,165,450,542$ \\
\hline & & CAATBOX1 & CAAT & + & 7 & $22,79,324,467,836,863,882$ \\
\hline & & WBOXATNPR1 & TTGAC & + & 1 & 252 \\
\hline & & DOFCOREZM & AAAG & + & 15 & $\begin{array}{l}7,93,97,164,175,197,230,284 \\
509,565,820,926,932,976\end{array}$ \\
\hline \multirow{5}{*}{21.} & \multirow{5}{*}{ AT3G61890 } & GATABOX & GATA & + & 2 & 631,650 \\
\hline & & WRKY71OS & TGAC & + & 1 & 253 \\
\hline & & CAATBOX1 & CAAT & + & 7 & $21,279,343,787,840,863,977$ \\
\hline & & WBOXATNPR1 & TTGAC & + & 1 & 353 \\
\hline & & DOFCOREZM & AAAG & + & 10 & $113,151,363,492,569,605,674,697,706,773$ \\
\hline \multirow{5}{*}{22} & \multirow{5}{*}{ AT2G01430 } & GATABOX & GATA & + & 4 & $61,252,402,680$ \\
\hline & & WRKY71OS & TGAC & + & 1 & 354 \\
\hline & & CAATBOX1 & CAAT & + & 4 & $386,619,765,884$ \\
\hline & & WRKY71OS & TGAC & + & 6 & $259,276,473,755,800,849$ \\
\hline & & DOFCOREZM & AAAG & + & 4 & $39,47,209,295$ \\
\hline \multirow{5}{*}{23} & \multirow{5}{*}{ AT5G03790 } & GATABOX & GATA & + & 5 & $160,352,409,680$ \\
\hline & & MYB1AT & WAACCA & + & 1 & 424 \\
\hline & & CAATBOX1 & CAAT & + & 6 & $43,282,363,613,690,868$ \\
\hline & & WRKY71OS & TGAC & + & 2 & 401,505 \\
\hline & & DOFCOREZM & AAAG & + & 11 & $68,100,186,383,461,634,804,810,988,992,996$ \\
\hline \multirow{5}{*}{24} & & GATABOX & GATA & + & 5 & $248,393,429,735,969$ \\
\hline & & MYB1AT & WAACCA & - & 2 & 55,166 \\
\hline & AT1G27045 & CAATBOX1 & CAAT & + & 7 & $234,314,362,475,787,830,971$ \\
\hline & & WRKY71OS & TGAC & - & 6 & $708,736,785,889,913,942$ \\
\hline & & DOFCOREZM & AAAG & + & 10 & $145,213,434,455,496,613,796,852,880,940$ \\
\hline & & GATABOX & GATA & + & 6 & $123,370,509,539,553,656$ \\
\hline & & MYB1AT & WAACCA & + & 2 & 841,846 \\
\hline 25 & AT1G70920 & CAATBOX1 & CAAT & + & 5 & $177,207,252,603,752$ \\
\hline & & WRKY71OS & TGAC & + & 5 & $122,137,613,704,863$ \\
\hline & & DOFCOREZM & AAAG & + & 6 & $1,47,90,381,494,874$ \\
\hline & & GATABOX & GATA & + & 9 & $38,84,106,181,214,367,371,497,936$ \\
\hline & & MYB1AT & WAACCA & + & 1 & 173 \\
\hline 26 & AT2G36610 & CAATBOX1 & CAAT & + & 3 & $49,588,772$ \\
\hline & & WRKY71OS & TGAC & + & 1 & 768 \\
\hline & & DOFCOREZM & AAAG & + & 8 & $219,255,344,876,901,943,972,986$ \\
\hline & & GATABOX & GATA & + & 8 & $23,101,109,349,763,873,879,964$ \\
\hline & & MYB1AT & WAACCA & + & 2 & 729,3 \\
\hline 27 & AT5G53980 & САAТВOX1 & CAAT & + & 7 & $227,257,262,279,771,791,942$, \\
\hline & & WRKY71OS & TGAC & + & 3 & $339,373,788$ \\
\hline & & DOFCOREZM & AAAG & + & 9 & $5,41,72,84,291,545,704,724,895$ \\
\hline & & GATABOX & GATA & + & 8 & $14,123,151,454,581,649,667,810$ \\
\hline & & MYB1AT & WAACCA & + & 1 & 652 \\
\hline 28 & AT1G79840 & CСАAТВOX1 & CAAT & + & 12 & $\begin{array}{l}268,293,400,516,542,726,787 \\
792,845,853,958,995\end{array}$ \\
\hline & & WRKY71OS & TGAC & + & 2 & 395,739 \\
\hline
\end{tabular}




\begin{tabular}{|c|c|c|c|c|c|c|}
\hline & & DOFCOREZM & AAAG & + & 5 & $432,526,633,667,797$ \\
\hline & & GATABOX & GATA & + & 5 & $219,454,491,612,940$ \\
\hline \multirow[t]{5}{*}{29} & AT1G05230 & CCAATBOX1 & CAAT & + & 9 & $9,47,368,711,754,876,938,963,973$ \\
\hline & & WRKY71OS & TGAC & + & 1 & 742 \\
\hline & & DOFCOREZM & AAAG & + & 7 & $51,58,65,101,666,691,805$ \\
\hline & & GATABOX & GATA & + & 4 & $241,344,510,738$ \\
\hline & & MYB1AT & WAACCA & - & 2 & 146,868 \\
\hline \multirow[t]{5}{*}{30} & AT4G00730 & CCAATBOX1 & CAAT & + & 6 & $27,152,420,424,679,983$ \\
\hline & & WRKY71OS & TGAC & + & 2 & 486,510 \\
\hline & & DOFCOREZM & AAAG & + & 19 & $\begin{array}{l}97,174,192,196,211,219,410, \\
465,614,703,721,725,867,884, \\
943,959,964,974\end{array}$ \\
\hline & & GATABOX & GATA & + & 2 & 185,351 \\
\hline & & MYB1AT & WAACCA & + & 1 & 847 \\
\hline \multirow[t]{5}{*}{31} & AT4G21750 & CCAATBOX1 & CAAT & + & 6 & $210,435,477$ \\
\hline & & WRKY71OS & TGAC & + & 3 & $424,824,863$ \\
\hline & & DOFCOREZM & AAAG & + & 7 & $68,176,392,573,772,809,854$ \\
\hline & & GATABOX & GATA & + & 2 & 196,368 \\
\hline & & MYB1AT & WAACCA & + & 2 & 527,592 \\
\hline \multirow[t]{5}{*}{32} & AT4G04890 & CCAATBOX1 & CAAT & + & 3 & $502,534,649$ \\
\hline & & WRKY71OS & TGAC & + & 6 & $105,141,219,654,699,873$ \\
\hline & & DOFCOREZM & AAAG & + & 5 & $176,247,349,849,924$ \\
\hline & & GATABOX & GATA & + & 6 & $43,289,296,426,455,913$ \\
\hline & & MYB1AT & WAACCA & - & 2 & 118,867 \\
\hline \multirow{5}{*}{33} & AT4G25530 & CCAATBOX1 & CAAT & + & 8 & $50,55,137,287,559,730,773,807$ \\
\hline & & WRKY71OS & TGAC & - & 2 & 48,654 \\
\hline & & DOFCOREZM & AAAG & + & 10 & $177,256,374,386,517,554,749,754,800,876$ \\
\hline & & GATABOX & GATA & + & 2 & 278,685 \\
\hline & & MYB1AT & WAACCA & + & 4 & $183,394,400,776$ \\
\hline \multirow[t]{5}{*}{34} & & CCAATBOX1 & CAAT & + & 5 & $48,113,202,534,686$ \\
\hline & AT4G32880 & WRKY71OS & TGAC & + & 2 & 718,743 \\
\hline & & DOFCOREZM & AAAG & + & 10 & $77,424,548,600.664,751,778,825,837,979$ \\
\hline & & GATABOX & GATA & + & 3 & $106,125,196$ \\
\hline & & MYB1AT & WAACCA & + & 1 & 109 \\
\hline \multirow[t]{4}{*}{35} & AT2G34710 & CCAATBOX1 & CAAT & + & 3 & $240,263,534$ \\
\hline & & WRKY71OS & TGAC & + & 1 & 234 \\
\hline & & DOFCOREZM & AAAG & + & 17 & $\begin{array}{l}41,119,127,133,167,272,277 \\
283,295,647,692,760,767,868,873,923,951\end{array}$ \\
\hline & & ARR1AT & NGATT & + & 3 & $297,710,934$ \\
\hline \multirow[t]{5}{*}{36} & AT3G61150 & CCAATBOX1 & CAAT & + & 4 & $160,272,363,583$ \\
\hline & & WRKY71OS & TGAC & + & 3 & $171,651,868$ \\
\hline & & DOFCOREZM & AAAG & + & 8 & $188,395,718,757,761,805,824,846$ \\
\hline & & ARR1AT & NGATT & + & 5 & $102,130,565,704,772$ \\
\hline & & AMYBOX1 & TAACARA & + & 1 & 623 \\
\hline \multirow[t]{4}{*}{37} & AT1G30490 & CCAATBOX1 & CAAT & + & 5 & $127,375,379,823,990$ \\
\hline & & WRKY71OS & TGAC & - & 2 & 142,587 \\
\hline & & DOFCOREZM & AAAG & + & 11 & $205,278,428,501,516,565,590,629,717,752,880$ \\
\hline & & ARR1AT & NGATT & + & 2 & 250,567 \\
\hline \multirow[t]{5}{*}{38} & AT1G52150 & CCAATBOX1 & CAAT & + & 7 & $289,502,577,603,671,683,782$, \\
\hline & & WRKY71OS & TGAC & + & 5 & $39,280,439,582,680$ \\
\hline & & DOFCOREZM & AAAG & + & 6 & $371,426,800,818,901,939$ \\
\hline & & ARR1AT & NGATT & + & 6 & $185,202,258,561,613,984$ \\
\hline & & AMYBOX1 & TAACARA & - & 1 & 17 \\
\hline \multirow[t]{5}{*}{39} & AT1G73360 & CCAATBOX1 & CAAT & + & 6 & $320,451,474,738,836,927$ \\
\hline & & WRKY71OS & TGAC & + & 1 & 337 \\
\hline & & DOFCOREZM & AAAG & + & 14 & $\begin{array}{l}85,143,169,518,594,612,732 \\
778,801,841,886,895,972,992\end{array}$ \\
\hline & & ARR1AT & NGATT & + & 3 & $95,211,637$ \\
\hline & & AMYBOX1 & TAACARA & + & 1 & 749 \\
\hline \multirow[t]{3}{*}{40} & AT2G32370 & CCAATBOX1 & CAAT & + & 5 & $276,455,725,841,995$ \\
\hline & & WRKY71OS & TGAC & + & 5 & $57,64,101,686,728$ \\
\hline & & DOFCOREZM & AAAG & + & 3 & $113,347,866$ \\
\hline
\end{tabular}




\begin{tabular}{|c|c|c|c|c|c|c|}
\hline & & ARR1AT & NGATT & + & 2 & 354,420 \\
\hline \multirow[t]{6}{*}{41} & AT5G52170 & CCAATBOX1 & CAAT & + & 1 & 875 \\
\hline & & WRKY71OS & TGAC & + & 4 & $141,479,872,970$ \\
\hline & & DOFCOREZM & AAAG & + & 13 & $\begin{array}{l}170,292,421,484,601,608,658 \\
692,718,723,773,815,865\end{array}$ \\
\hline & & ARR1AT & NGATT & + & 4 & $622,963,804,809$ \\
\hline & & ARR1AT & & & & \\
\hline & & AMYBOX1 & TAACARA & + & 1 & 714 \\
\hline \multirow[t]{5}{*}{42} & AT5G60690 & CCAATBOX1 & CCAAT & - & 2 & 535,786 \\
\hline & & WRKY71OS & TGAC & + & 1 & 606 \\
\hline & & DOFCOREZM & AAAG & + & 5 & $127,726,818,839,941$ \\
\hline & & GATABOX & GATA & + & 8 & $376,430,458,488,505,744,794,902$ \\
\hline & & MYBCORE & CNGTTR & + & 1 & 448 \\
\hline \multirow[t]{5}{*}{43} & AT1G17920 & CCAATBOX1 & CCAAT & - & 2 & 172,445 \\
\hline & & WRKY71OS & TGAC & + & 3 & $372,491,674$ \\
\hline & & DOFCOREZM & AAAG & + & 11 & $179.191,243,274,482,514.547,735,889.898,950$ \\
\hline & & GATABOX & GATA & + & 5 & $87.96 .226,277,935$ \\
\hline & & MYBCORE & CNGTTR & + & 2 & 570,814 \\
\hline \multirow[t]{5}{*}{44} & AT5G46880 & CCAATBOX1 & CCAAT & -- & -- & -- \\
\hline & & WRKY71OS & TGAC & + & 3 & $182,247,271$ \\
\hline & & DOFCOREZM & AAAG & + & 12 & $43,66,241,464,422,490,495,607,610,631,637,880$ \\
\hline & & GATABOX & GATA & + & 1 & 822 \\
\hline & & MYBCORE & CNGTTR & + & 4 & $221,396,409.915$ \\
\hline \multirow[t]{5}{*}{45} & AT4G17710 & CCAATBOX1 & CCAAT & + & 1 & 486 \\
\hline & & WRKY71OS & TGAC & + & 2 & 275,416 \\
\hline & & DOFCOREZM & AAAG & + & 11 & $56,87,352,426,499,593,710,750,928,935,951$ \\
\hline & & GATABOX & GATA & + & 5 & $59,98,146,355,368$ \\
\hline & & MYBCORE & CNGTTR & + & 3 & $782,790,803$ \\
\hline \multirow[t]{5}{*}{46} & AT3G03260 & CCAATBOX1 & CCAAT & + & 1 & 692 \\
\hline & & WRKY71OS & TGAC & + & 4 & $496,536,543,657$ \\
\hline & & DOFCOREZM & AAAG & + & 5 & $26,220,414,526,706$ \\
\hline & & GATABOX & GATA & + & 2 & 265,457 \\
\hline & & MYBCORE & CNGTTR & + & 1 & 192 \\
\hline \multirow[t]{5}{*}{47} & AT5G17320 & CCAATBOX1 & CCAAT & - & 1 & 157 \\
\hline & & WRKY71OS & TGAC & + & 3 & $383,447,876$ \\
\hline & & DOFCOREZM & AAAG & + & 9 & $98,362,405,470,491,572,629,733,748$ \\
\hline & & GATABOX & GATA & + & 3 & $311,440,718$ \\
\hline & & MYBCORE & CNGTTR & + & 1 & 70 \\
\hline \multirow[t]{5}{*}{48} & AT1G34650 & CCAATBOX1 & CCAAT & + & 1 & 612 \\
\hline & & WRKY71OS & TGAC & + & 4 & $529,603,628,876$ \\
\hline & & DOFCOREZM & AAAG & + & 3 & $372,376,397$ \\
\hline & & GATABOX & GATA & + & 3 & $255,682,977$ \\
\hline & & MYBCORE & CNGTTR & + & 1 & 78 \\
\hline
\end{tabular}

